\title{
SIMULATION MODELING OF INTERPLANETARY SHOCKS ARRIVAL TIME PREDICTION ON HISTORICAL DATA SET
}

\author{
Volodymyr Turchenko, Viktor Demchuk, Anatoly Sachenko
} Research Institute of Intelligent Computer Systems, Ternopil National Economic University,
Peremoga Square 3, 46004, Ternopil, Ukraine, \{vtu, vde, as\}@tanet.edu.te.ua

\begin{abstract}
An approach to prediction of the arrival time of interplanetary shocks using neural networks based on the data gathered from single EPAM (Electron, Proton and Alpha Monitor) channel of NASA's ACE (Advanced Composition Explorer) spacecraft is proposed in this paper. A short description of ACE spacecraft and the data, published online on the appropriate web-site, are considered. A data choice to fulfill a prediction of interplanetary shocks is proven and structure of neural network is described. The results of simulation modeling in MATLAB are considered in the end of the paper.
\end{abstract}

Keywords: Space weather, solar wind, ACE spacecraft, predicting interplanetary shocks, neural networks.

\section{INTRODUCTION}

Interplanetary shocks in the space are the regions created by supersonic gas flow with sharp differences of gas density, pressure, temperature, ionization and other its parameters [1]. The solar wind, putting this gas to the Earth, goes to the Earth's magnetosphere at about $500 \mathrm{~km} / \mathrm{s}$ and makes a shock due to a resistance of Earth's magnetic field. The energetic storm particle (ESP) events are associated with interplanetary shocks passages and close related to the geomagnetic storms. Both these events negatively influence on spacecrafts and satellites on a low-orbital Earth's orbit, terrestrial high-frequency radio communications and radars, electrical grids and electrical power systems, and people's health [2]. For example, GOES-7 weather satellite lost half of its solar cells during a large proton release by the sun during the powerful March 13, 1989 storm which cut the operating life span of this satellite in half. ANIK E-1 and E-2 (January 2021, 1994) two Canadian communications satellites were disabled due to the elevated activity of highenergy electrons in the magnetosphere. On January 11, 1997 AT\&T experienced a massive power failure in its Telstar 401 satellite [3]. There are much more examples of satellites lose and their temporal disabling caused by the interplanetary shocks. Therefore there are urgent tasks to predict the solar activity and its influence on Earth's magnetosphere and the time of interplanetary shocks arrival and peak intensity of energetic particles traveling with the solar wind.
During last decades many strategies were proposed for space weather prediction based on the data comes from satellites and terrestrial observatories. Many research teams use neural network approach for space weather prediction. R. A. Calvo and H. A. Ceccatto use feed-forward neural networks to study the solar dynamics, as measured by the annual mean value of the Wolf number. They conclude that neural networks are a reliable tool for time series analysis. In particular, they seem to be able to capture the intrinsic dynamics of solar activity, producing good longterm forecastings for periods of at least a complete solar cycle [4]. A. Dmitriev and Yu. Minaeva et al. use recurrent ANNs for modeling of self-consistent time series of geomagnetic indexes Dst, Kp, AP, etc [5]. Z. Voros and D. Jankovicova propose prediction of geomagnetic activity based on a method using local Holder exponents $a$. The backpropagation artificial neural network model with feedback connection was used for the study of the solar wind magnetosphere coupling and prediction of geomagnetic Dst index [6].

J. Vandegriff et al. [7] have developed an algorithm that can forecast the arrival of ESP events. The authors use historical ion data from the NASA's Advanced Composition Explorer (ACE) spacecraft, which is stationed in a halo orbit around Lagrange point L1 at the distance about 1.5 million $\mathrm{km}$ from the Earth. They trained an artificial neural network to detect the characteristic signals that warn of an impending event. The network predicts the time 
remaining until the maximum intensity of the ions is reached on the Earth. For the input of the prediction model they have used five ion channels (P1, P3, P5, P6, P7) provided by the web-site of NOAA (U.S. National Oceanic and Atmospheric Administration) real-time system and additional derivative parameters. However the choice of these data is not quite well explained and the average uncertainty of the prediction by the proposed method is 8.9 hours at 24-hours time interval.

The goal of this paper is to estimate usage of separate ACE channels for prediction of the interplanetary shocks arrival time in order to decrease a computational complexity of a prediction algorithm and the relative prediction error of interplanetary shocks arrival time.

\section{ACE/EPAM DATA SET AND PREDICTION APPROACH}

The ACE Electron, Proton, and Alpha Monitor (EPAM) data can characterize the dynamic behavior of electrons and ions with $\sim 0.03$ to $\sim 5 \mathrm{MeV}$ that are accelerated by impulsive solar flares and by interplanetary shocks associated with Coronal Mass Injections. EPAM instrument includes two telescope assemblies with five separate apertures. The telescopes use the spin of the spacecraft to sweep the full sky. Solid-state detectors are used to measure the energy and composition of the incoming particles. The eight channels from the EPAM/LEMS30 (Low-Energy Magnetic Spectrometer) detector and their energy passbands [8] are presented in Table 1.

Table 1. Energy passbands of LEMS30/ACE detector

\begin{tabular}{|c|c|c|}
\hline Energy Channel & $\begin{array}{c}\text { Passband } \\
(\mathbf{M e V})\end{array}$ & Species \\
\hline P1 & $0.047-0.065$ & Ions \\
\hline P2 & $0.065-0.112$ & Ions \\
\hline P3 & $0.112-0.187$ & Ions \\
\hline P4 & $0.187-0.310$ & Ions \\
\hline P5 & $0.310-0.580$ & Ions \\
\hline P6 & $0.580-1.06$ & Ions \\
\hline P7 & $1.06-1.91$ & Ions \\
\hline P8 & $1.91-4.75$ & Ions \\
\hline
\end{tabular}

ACE browse data are designed for monitoring large scale particle and field behavior and for selecting interesting time periods. The data are automatically generated from the spacecraft data stream using simple algorithms provided by the instrument investigators and published on the web by NOAA in real-time. We used ACE Level 2 LEMS30 detector historical data that is suitable for a scientific research [9].

Interplanetary shock events can be recognized from the steam of EPAM data using two criteria [7]: velocity dispersion in the shock onset and a peak intensity greater than $10^{5}$ particles/(s $\mathrm{cm}^{2}$ ster $\left.\mathrm{keV}\right)$ for the 47-65 keV proton channel (see channel P1 in Table 1). J. Vandegriff et al. [7] have used a simple trigger designed to detect velocity dispersion in order to detect the onset. The trigger examines such additional parameters as the spectral slope, the average height of the energy spectrum, and the time derivatives of these quantities. All mentioned quantities are used for neural network training, in particularly the five ion channels (P1, P3, P5, P6, P7) provided by the NOAA real-time system, which are listed in Table 1 and the five quantities mentioned above, an anisotropy coefficient, spectral slope (SS), intensity midpoint (IMP) and time derivatives of these quantities (SS' and IMP'). Therefore a neural network had ten inputs and one output, describing the time before shock arrival, i.e. the time then ion intensity became greater $10^{5}$ particles/(s $\mathrm{cm}^{2}$ ster $\left.\mathrm{keV}\right)$. However such approach does not effectively use a prediction model since each time before arrival should correspond to the input part of the appropriate training vector. Practically this approach leads to necessity having an input data for neural network at each prediction step and therefore it is not possible to provide longterm prediction using this model.

In order to test our approach we have used two shock events similarly to [7]:

- event 1 - onset begin at $14.00,248$ days in 2000; shock begin at 12.00, 250 day of 2000 (06/09/2000) and duration of this event is 46 hours (550 points of 5-minute averaged solar particle fluxes);

- event 2 - onset begin at 0.00, 21 day of 2001; shock begin at $6.00,23$ day of 2001 $(23 / 01 / 2001)$ and duration of this event is 30 hours (360 points of 5-minute averaged solar particle fluxes).

The graphs of EPAM solar particle fluxes of each channel P1-P8 for the event 1 are shown on Fig. 1. There are just an example figures, similar intensities are available for other shock events. A numerical analysis of graphs shown, that only P1 and P2 channels can provide a peak intensity greater than $10^{5}$ particles/(s $\mathrm{cm}^{2}$ ster $\left.\mathrm{keV}\right)$. Therefore it is possible to use the data from at least one channel for prediction the time before shock arrival. Within our prediction method we are going to predict an intensity excess of $10^{5}$ particles/(s $\mathrm{cm}^{2}$ ster $\left.\mathrm{keV}\right)$ on the time interval. The moment of time when the intensity will be greater that $10^{5}$ particles/ $\left(\mathrm{s} \mathrm{cm}^{2}\right.$ ster $\mathrm{keV}$ ) is treated as a predicted moment of interplanetary shock. A comparison with a real time of appropriate EPAM data is considered as relative error of interplanetary shock arrival time. Other channels P4-P8 except for P3 channel could be used for more precise estimation of onset moment. Free 
EPAM 1-minute and 5-minute data are accessible on anonymous FTP server [10]. The data are putted on the server each hour with a delay of 7 minutes which allows providing prediction in real time.

\section{STRUCTURE OF NEURAL NETWORK}

It is expediently to use a multi-layer perceptron to fulfill the prediction task, since this architecture has the advantage of being simple and widely used for prediction tasks [11-12].

The output value of three-layer perceptron (Fig. 2) can be formulated as:
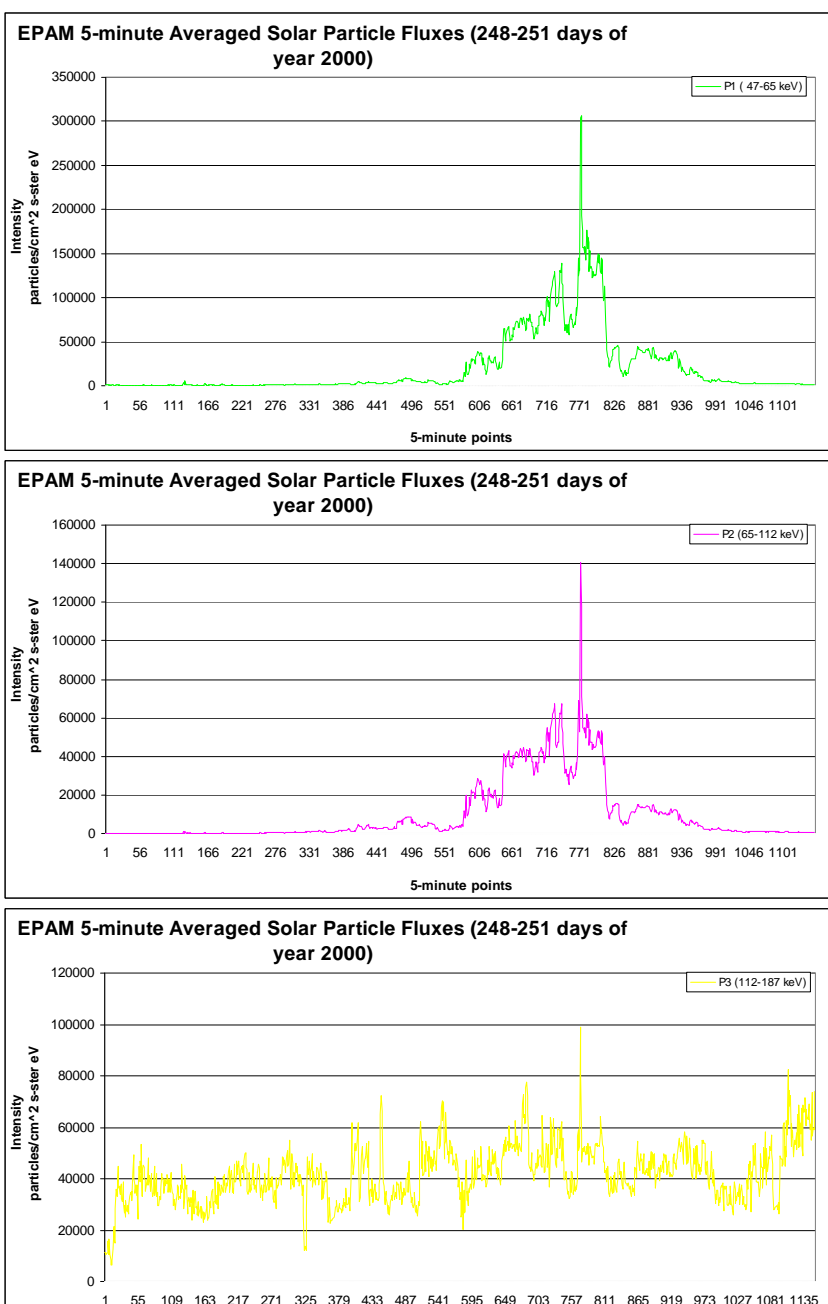
5-minute points

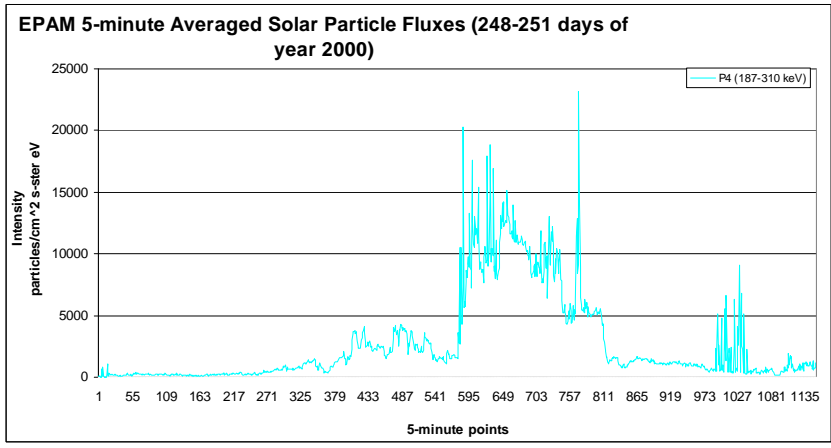

$$
y=F_{3}\left(\sum_{i=1}^{N} w_{i 3} h_{i}-T\right),
$$

where $N$ is the number of neurons in the hidden layer, $w_{i 3}$ is the weight of the synapse from neuron $i$ in the hidden layer to the output neuron, $h_{i}$ is the output of neuron $i, T$ is the threshold of the output neuron and $F_{3}$ is the activation function of the output neuron.
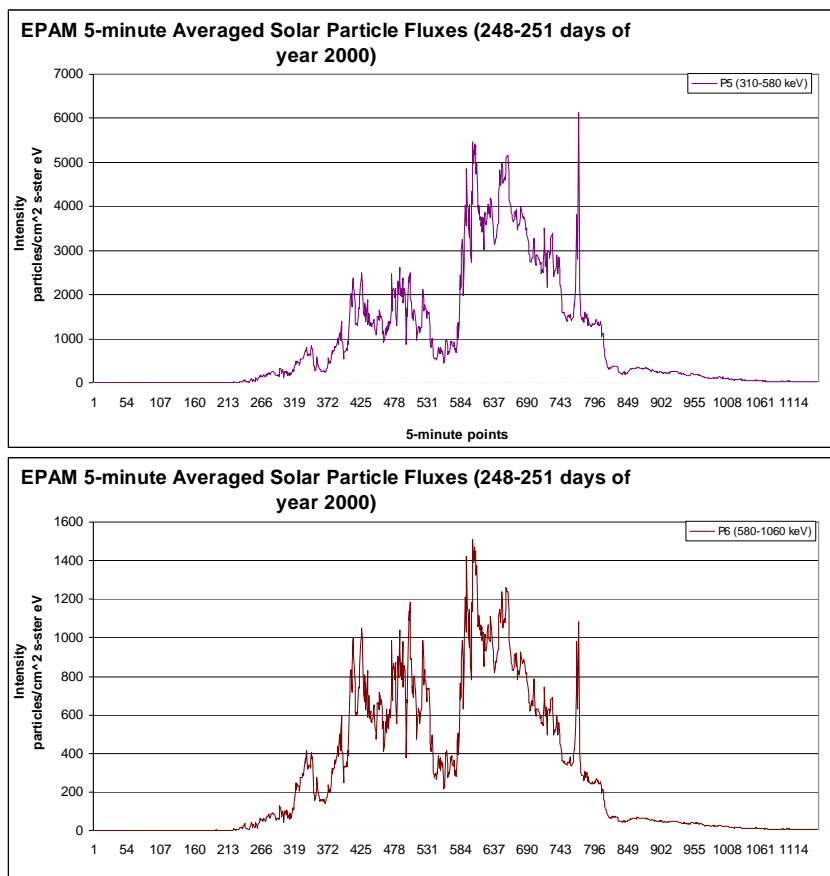

5-minute points

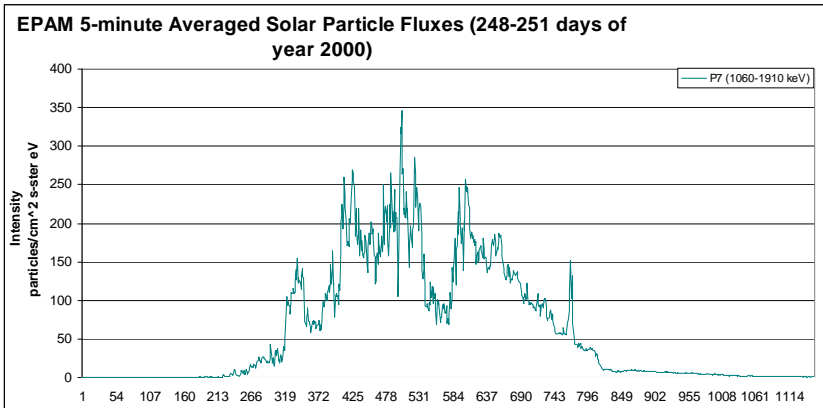
5-minute points

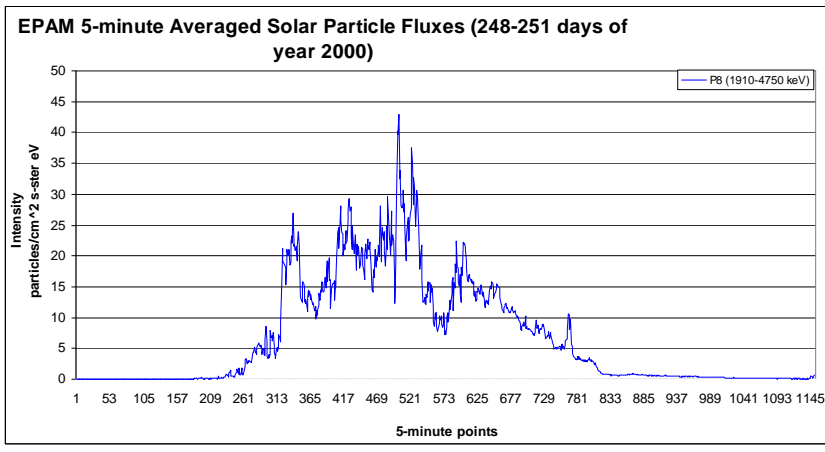

Fig. 1 - Particle intensities in separate channels P1-P8 for historical data test sequence: 248-251 days of year 2000 
The output value of neuron ${ }^{j}$ in the hidden layer is given by:

$$
h_{j}=F_{2}\left(\sum_{i=1}^{M} w_{i j} x_{i}-T_{j}\right),
$$

where $w_{i j}$ are the weights from the input neurons to neuron ${ }^{j}$ in the hidden layer, ${ }^{x_{i}}$ are the input values and $T_{j}$ is the threshold of neuron ${ }^{j}$. The logistic activation function is used for the neurons of the hidden layer and the linear activation function, having a coefficient $k$, is used for the output neuron.

The Levenberg-Marquardt algorithm is used for the training since it appears to be the fastest method for training moderate-sized feed forward neural networks [13].

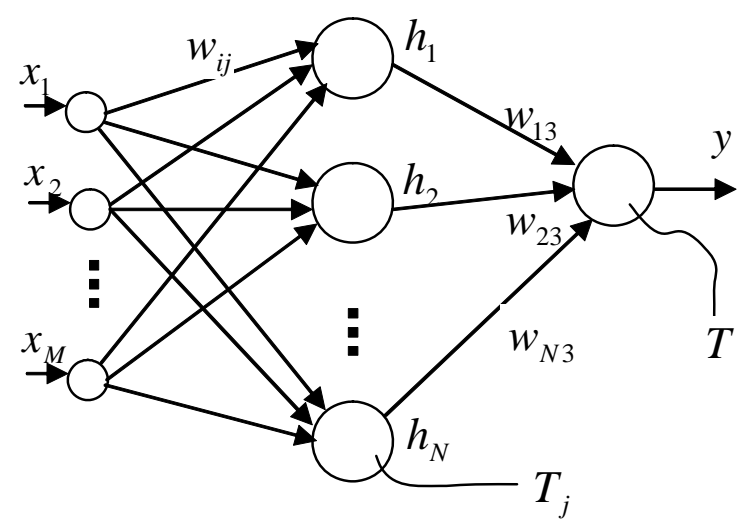

Fig. 2 - Structure of neural network

\section{SIMULATION MODELLING RESULTS}

An experimental simulation modeling has been done in the MATLAB environment [14]. An input training set has been formed according to BoxJenkins [15] method. The size of input window we have chosen to be equal to 5 , the size of the output window is equal to one since we are going to predict one step-by-step value of particle intensity and estimate when it will be greater than $10^{5}$ particles/(s $\mathrm{cm}^{2}$ ster $\mathrm{keV}$ ). The multi-layer perceptron with 5 input neurons, 5 hidden neurons with tangent activation function and 1 output linear neuron has been used for prediction. We have used a Levenberg-Marquardt method for perceptron training till sum-squared error (SSE) of $10^{-3}$. The results of simulation modeling fulfilled several times for each shock event are placed below.

The prediction result of energetic particles intensity for the event $1(06 / 09 / 2000)$ is depicted on Fig. 3. The 550 five-minute data set is used for perceptron's training and the same data are used for prediction in order to estimate a relative prediction error inside the training set. As it is seen the predicted and real data are practically the same. The analysis of the numerical data of the result has shown that the predicted time of shock arrival is equal to 540 value from onset and real time of shock arrival is equal to 545 value from onset. Therefore the relative prediction error inside the training set is less than $0.01 \%$.

Then, the perceptron trained on event 1 (550 data points) has been used to predict the shock arrival for the event 2 (23/01/2001) with length of 360 data points. As it is seen from Fig. 4, the predicted and real intensities are practically the same too. The analysis of numerical data shown, that the predicted time of shock arrival is equal to 352 value from onset and real time of shock arrival is equal to 355 value from onset. Therefore in this case the relative prediction error outside the training set is less than $0.01 \%$ too. The prediction result for the event 2 by the perceptron trained on the reduced data set (360 values) from the event 1 is depicted on Fig. 5.

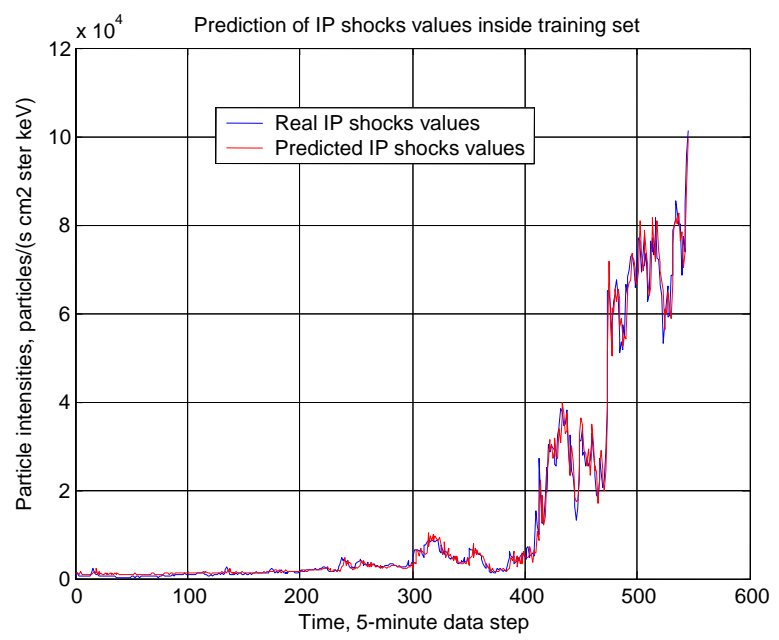

Fig. 3 - Prediction interplanetary shocks for event 1 with $\mathbf{5 5 0}$ data in the training set

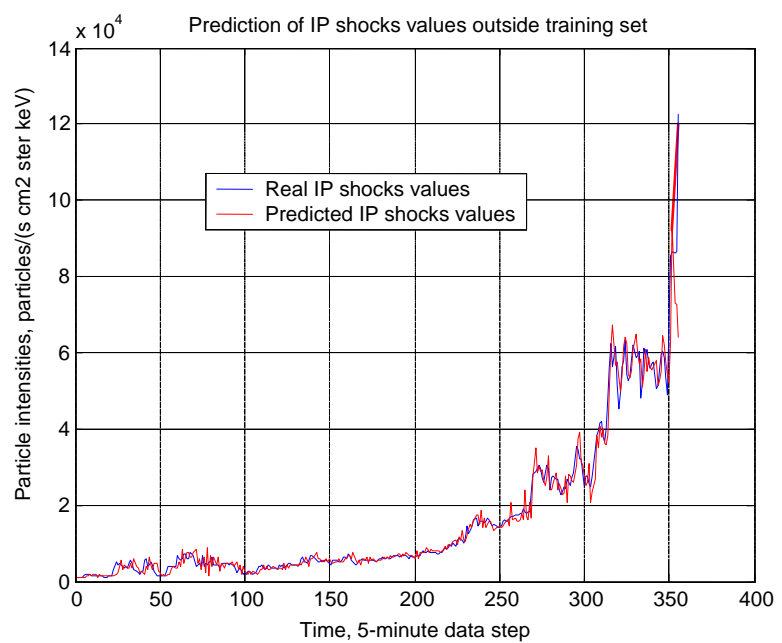

Fig. 4 - Prediction interplanetary shocks for event 2 with 550 data in the training set 


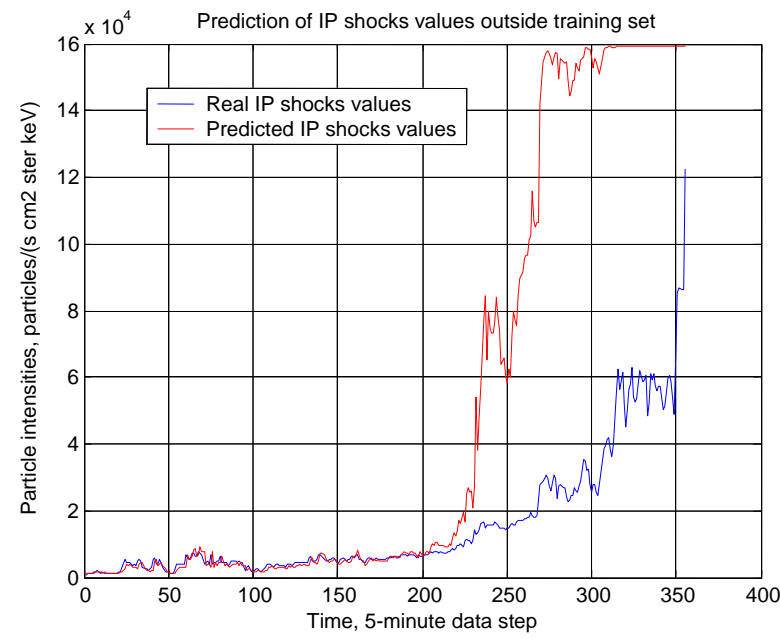

Fig. 5 - Prediction interplanetary shocks for event 2 with 360 data in the training set

The analysis of numerical data shown that the predicted time of shock arrival is equal to 263 value from onset and real time of shock arrival is equal to 355 value from onset. Therefore the relative prediction error of interplanetary shock arrival is about $27 \%$ at reduced training set for perceptron training.

\section{CONCLUSIONS}

An approach to interplanetary shocks arrival time prediction is proposed in this paper based on the usage of separate channel's EPAM data of ACE spacecraft. Neural based approach is tested using energetic particle intensities for the range 47-65 $\mathrm{keV}$. The data about interplanetary shock 06/09/2000 are used for neural network training and the data about interplanetary shock 23/01/2001 are used for the testing. Experimental simulation modeling results have shown non-stability of the prediction changing in relative prediction error from accurate $0.01 \%$ to not quite accurate $27 \%$ gathered on reduced training set. Therefore in future investigations it is expedient to fulfill a series of experimental researches on usage both channels P1 and P2 of EPAM data for the prediction and test both approaches on wide set of interplanetary shocks events.

\section{ACKNOWLEDGEMENTS}

This research is supported by STCU (www.stcu.int) grant \#3872 "Development of effective GRID technologies for ecology monitoring using satellite data" (2005-2007). This support is gratefully acknowledged.

\section{REFERENCES}

[1] http://www-spc.igpp.ucla.edu/ newbury/papers /sw electrons/node9.html
[2] A. Joselyn, J. Anderson, H. Coffey et al.., Solar Cycle 23 Project: Summary of Panel Findings, Short Reference Paper, Nov. 1996.

[3] Solar Storms: The Silent Menace, by Dr. Sten Odenwald, 1998.

http://image.gsfc.nasa.gov/poetry/workbook/ storms.html

[4] R. A. Calvo, H. A. Ceccatto and R. D. Piacentini. Neural network prediction of solar activity, Astrophysical Journal, Part 1, 444 (2), 1995, pp. 916-921

[5] A. Dmitriev, Yu. Minaeva, Yu. Orlov, M. Riazantseva, I. Veselovsky. Solar Activity Forecasting on 1999-2000 by Means of Artificial Neural Networks, Reported on EGS XXIV General Assembly, The Hague, The Netherlands 22 April 1999.

[6] Z. Voros and D. Jankovicova. Neural network prediction of geomagnetic activity: a method using local Holder exponents, Nonlinear Processes in Geophysics 9, 2002, pp. 425-433.

[7] J. Vandegriff, K. Wagstaff, G. Ho, J. Plauger. Forecasting space weather: Predicting interplanetary shocks using neural networks, Advances in Space Research 36, 2005, pp. 2323-2327.

[8] http://www.srl.caltech.edu/ACE/ASC/level2/epa m_l2desc.html

[9] http://www.srl.caltech.edu/ACE/ASC/level2/lvl 2DATA_EPAM.html

[10] http://sec.noaa.gov/ftpmenu/lists/ace.html

[11]K. Hornik, M. Stinchcombe, H. White. Multilayer Feedforward Networks are Universal Approximators, Neural Networks 2, 1989, pp. 359-366.

[12]V. Golovko. Neural Networks: training, models and applications. Radiotechnika. Moscow, 2001. pp. 256 (in Russian).

[13] M.T. Hagan, M. Menhaj. Training feed-forward networks with the Marquardt algorithm, IEEE Transactions on Neural Networks 5 (6), 1994, pp. 989-993.

[14] http://www.mathworks.com/

[15]G. Box, G. Jenkins. Time Series analysis: Forecasting and Control. Holden-Day. San Francisco, 1976.

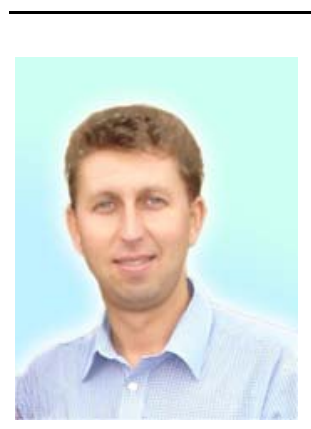

Volodymyr Turchenko was born in 1973, in Ternopil, Ukraine. He received his Engineer Diploma in systems engineering from Brest Polytechnic Institute, Brest, Belarus (1995) and his Ph.D. degree in computer engineering from National University "Lviv Polytechnics", Lviv, Ukraine 
(2001). Now he is Associate Professor of the Information Computing System and Control Department of the Faculty of Computer Information Technologies, Ternopil State Economic University (TSEU), Ternopil, Ukraine. He is a leader of Neural Network and Parallel Computing Research Team within the Research Institute of Intelligent Computer Systems of TSEU. He has published more than 60 scientific papers. His main research interests are Neural Networks, Parallel Computing and Intelligent Distributed Sensor Networks.

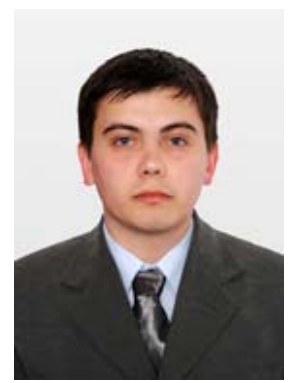

Viktor Demchuk from 2002 year student of Faculty of Computers Information Technologies of the Ternopil National Economic University. From 2004 year works as the technician of Educational Computer Laboratory in Department of the Informative Computer Systems and Management.

Now he is an engineer of International Scientific Journal of Computing. Areas of scientific interests: Neural Networks, Parallel Algorithms, High Performance Computing, Grid.
Anatoly Sachenko is Professor and Head of the Department of Information Computing Systems and Control and Director of American- Ukrainian Program in Computer Science, Ternopil State Economic University.

He earned his B.Eng. Degree in Electrical Engineering at L'viv Polytechnic Institute in 1968 and his PhD Degree in Electrical Engineering at L'viv Physics and Mechanics Institute in 1978 and his Doctor of Technical Sciences Degree in Electrical and Computer Engineering at Leningrad Electrotechnic Institute in 1988. Since 1991 he has been Honored Inventor of Ukraine, since 1993 he has been IEEE Senior Member.

His main Areas of Research Interest are Implementation of Artificial Neural Network, Distributed System and Network, Parallel Computing, Intelligent Controllers for Automated and Robotics Systems. He has published over 300 papers in areas above. 